\title{
Ever HRD a ubiquitin-gated channel?
}

\author{
Cell Research (2016) 26:1075-1076. doi:10.1038/cr.2016.92; published online 5 August 2016
}

\begin{abstract}
Elimination of misfolded proteins of the endoplasmic reticulum (ER) requires their retrotranslocation from the ER to the cytosol via membranebound ubiquitin ligase complexes. Baldridge and Rapoport now reconstitute a key step of retrotranslocation, demonstrating a protein conduit gated by ubiquitination.
\end{abstract}

In the endoplasmic reticulum (ER), the fidelity of protein synthesis is safeguarded by ER-associated protein degradation (ERAD). This pathway retrotranslocates aberrant polypeptides from the ER to the cytosol for degradation by the proteasome [1]. In S. cerevisiae, several membrane-embedded ubiquitin ligases are responsible for ubiquitination of retrotranslocated products: Hrd1 ubiquitinates substrates bearing misfolded domains in the lumen and membranes; Doa10 deals with substrates carrying a misfolded cytosolic domain [2,3]. A key, yet poorly-defined step in ERAD is the translocation of polypeptide across the membrane, postulated to occur through conduits formed by ubiquitin ligases such as Hrd1 [4]. In a recent issue of Cell, Baldridge and Rapoport demonstrate that $\mathrm{Hrd} 1$ is indeed a protein retrotranslocation channel [5].

To reconstitute a reaction that simulates closely the retrotranslocation process, Baldridge generated CPY* variants carrying a transmembrane segment from either Pdr5 or Spt23 (referred to as CPY*-TMs). Similar to CPY*[6], CPY*-TMs were degraded by Hrd1 in cells, and in vitro, only CPY*-TMs, but not the folded counterparts could be efficiently ubiquitinated by $\mathrm{Hrd} 1$ in detergent. Importantly, CPY*-TMs could be efficiently incorporated into proteoliposomes notwithstanding that only $\sim 50 \%$ of the proteins have the desired orientation (the $\mathrm{CPY}^{*}$ domain in the lumen). Because only ubiquitination of CPY*-TMs with CPY* initially located in the lumen by ubiquitination machinery on the outside can be taken as an indication of retrotranslocation, the authors developed a sortase-based method that labeled substrates of different topologies with distinct colors. They then incubated CPY*-TM-containing vesicles with Hrd1 in the presence of low concentrations of the detergent, decyl maltose neopentyl glycol (DMNG). This treatment resulted in the incorporation of $\mathrm{Hrd} 1$ into these vesicles in a uniform orientation with the catalytic RING (really interesting gene) domain facing the outside. When the vesicles were further incubated with ATP and ubiquitination factors, substrates of both topologies were polyubiquitinated, but only the luminally-oriented CPY*-TM population requires the Hrd1 transmembrane segments for ubiquitination. Because the reactions contain only Hrd1 and CPY*-TMs, Hrd1 apparently can move at least a portion of the luminally-localized CPY*-TMs across the membrane using a conduit formed by its transmembrane domain.

The ubiquitination-based retrotranslocation assay is elegant, but it cannot gauge the extent of retrotranslocation since a small retrotranslocated segment may be sufficient for ubiquitination. The authors therefore established another assay using CPY*-TMs with Alexa488 labels on lysine residues throughout the $\mathrm{CPY}^{*}$ domain. They incorporated these substrates into proteoliposomes and quenched the labels on CPY* facing the outside with Alexa488 antibodies before initiating retrotranslocation. During the incubation, additional Alexa488 antibodies were included, thus any labels on a $\mathrm{CPY}^{*}$ domain emerging from the lumen as a result of retrotranslocation would be quenched. Consistent with the ubiquitination-based assay, they observed a time-dependent loss in fluorescence that requires ATP and the catalytic activity of Hrd1. Because multiple fluorophores carried by CPY*TMs can be quenched significantly, it seems that Hrd1 by itself can translocate a significant portion of CPY* in a ubiquitination-dependent manner.

Baldridge noticed that during the reaction both CPY*-TMs and Hrd1 were ubiquitinated on lysine residues. To test whether ubiquitination of CPY*-TM is essential for retrotranslocation, they generated a lysine-free CPY*-TM variant. As anticipated, polyubiquitination of lysineless CPY*-TM in vitro by Hrd1 was drastically reduced. Nonetheless, it was retrotranslocated normally as the lysine-containing counterpart, suggesting that substrate ubiquitination is dispensable for retrotranslocation.

Finally, to test whether autoubiquitination of Hrd1 is critical, the authors substituted lysine residues in different parts of Hrd1 with arginine. Removal of lysine from either the transmembrane domain or a cytosolic segment juxtaposing the RING had no effect, whereas lysine substitutions in the RING domain impaired CPY*-TM retrotranslocation without affecting Hrd1's ligase activity. Additional experiments identified lysine 373,387 , and 407 as the critical residues for the retrotranslocation activity in vitro and Hrd1 function in cells. Immunoprecipitation showed that a fraction of endogenous $\mathrm{Hrd} 1$ was 


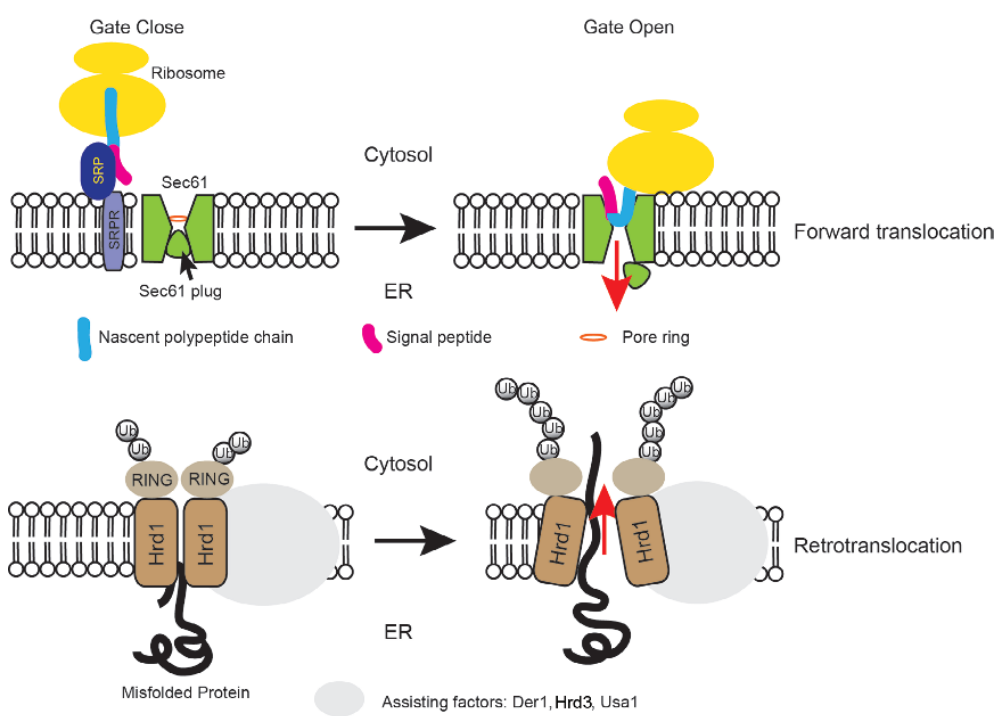

Figure $1 \mathrm{~A}$ comparison of the channel gating mechanisms for forward and retrotranslocation at the ER. Top: A model for Sec61-mediated forward translocation based on structural and biochemical studies on bacterial SecY [10]. A monomeric Sec61 complex forms a channel that is constricted at the center by a pore ring formed by a collection of hydrophobic residues. A helix 'plug' further seals the channel from the luminal side. When a nascent polypeptide bearing a signal sequence is synthesized by the ribosome, the signal sequence is captured by the signal recognition particle (SRP), which brings the nascent chain-ribosome complex to the Sec61 channel via an ER-anchored SRP receptor. Nascent chain is inserted into the channel as a loop. The interaction of the signal sequence with the channel causes a conformational change, resulting in the removal of the 'plug' and pore opening. Bottom: Hrd1-mediated retrotranslocation for ERAD-L substrates. Hrd1 forms a homooligomerized pore in the ER membrane. Substrate binding to Hrd1 in the lumen causes autoubiquitination of the RING domain in the cytosol, which in turn activates the channel and allows substrate to slip through the membrane. In cells, Hrd1 is assisted by cofactors that may coordinate substrate binding with channel activation.

ubiquitinated on these lysine residues in cells. Taken together, these results strongly indicate that retrotranslocation by Hrd1 involves activation of Hrd1 by autoubiquitination. Based on the observation that ubiquitin conjugates on Hrd1 increase its affinity to CPY*-TMs without changing its oligomerization state, the authors postulate that ubiquitination may alter Hrd 1 conformation to serve a gate-opening function.

Over the years, the identity of retrotranslocation channel has been a major controversial issue, therefore, the establishment of Hrd1 as a retrotranslocation conduit is clearly a milestone in ERAD research. The proposed channel gating mechanism is an exciting and unprecedented idea since for protein translocation processes characterized to date, channel opening is usually gated by substrate binding (Figure 1). Because the Hrd1 RING domain is localized in the cytosol, the model implies the existence of a coupling mechanism that links substrate binding in the lumen to Hrd1 autoubiquitination in the cytosol. In cells, Hrd1-interacting proteins such as $\mathrm{Hrd} 3$ may fulfill such a function by inhibiting Hrd1 autoubiquitination until substrate arrival. Retrotranslocation may be accompanied by rapid deubiquitination of Hrd1 to ensure its structural integrity. This is consistent with the implication of several deubiquitinases in both yeast and mammalian ERAD [7].

At the moment, it is unintuitive to conceive a straightforward mechanism whereby autoubiquitination of a soluble domain can influence the conformation of a lipid-embedded channel. Autoubiquitination, as a hallmark of RINGbearing ubiquitin ligases, was known for years [8], but only recently researchers found that autoubiquitination can have a positive function other than destabilizing the modified ligase. This often requires a cofactor that use a ubiquitinbinding domain to 'decipher' the ubiquitin signal on ligase [9]. Thus, the transmembrane segments of Hrd1 may collectively form a ubiquitin-binding site on the ER surface. Alternatively, ubiquitin conjugates may allosterically alter the conformation of the Hrd1 transmembrane segments. Further elucidation of this gating mechanism requires determination of Hrdl structures in unmodified and modified states, which would have been a huge challenge in the past. Fortunately, with the recent advancement of the high-resolution electron microscopy technology, we may not need to wait long for an answer.

\section{Ting Zhang ${ }^{1}$, Yihong $\mathrm{Ye}^{1}$ \\ ${ }^{l}$ Laboratory of Molecular Biology, National Institute of Diabetes and Digestive and Kidney Diseases, National Institutes of Health, Bethesda, MD 20892, USA \\ Correspondence: Yihong Ye \\ Tel: +1-301-5940845; Fax: +1-301-4960201 \\ E-mail: yihongy@mail.nih.gov}

\section{References}

1 Christianson JC, Ye Y. Nat Struct Mol Biol 2014; 21:325-335.

2 Vashist S, Ng DT. J Cell Biol 2004; 165:4152.

3 Carvalho P, Goder V, Rapoport TA. Cell 2006; 126:361-373.

4 Carvalho P, Stanley AM, Rapoport TA. Cell 2010; 143:579-591.

5 Baldridge RD, Rapoport TA. Cell 2016; 166:394-407.

6 Stein A, Ruggiano A, Carvalho P, et al. Cell 2014; 158:1375-1388.

7 Liu Y, Ye Y. Curr Protein Pept Sci 2012; 13:436-436.

8 Lorick KL, Jensen JP, Fang S, et al. Proc Natl Acad Sci USA 1999; 96:11364-11369.

9 Yau R, Rape M. Nat Cell Biol 2016; 18:579586.

10 Osborne AR, Rapoport TA, van den Berg B. Annu Rev Cell Dev Biol 2005; 21:529-550. 